Estudios sobre el Mensaje Periodístico ISSN-e: 1988-2696

http://dx.doi.org/10.5209/ESMP.55606

\title{
La enseñanza del protocolo y la organización de eventos en el espacio europeo de educación superior: análisis comparado de los planes de estudio
}

\author{
Julio César Herrero ${ }^{1}$; María del Mar Perelló Roselló ${ }^{2}$
}

Recibido: 28 de enero de 2016 / Aceptado: 22 de julio de 2016

Resumen. El estudio del protocolo y la organización de eventos es muy reciente en España en el ámbito universitario. Tan sólo cinco universidades lo ofertan. Sin embargo, se trata de una formación con amplia tradición superior en el resto de Europa y del mundo. Este artículo analiza la situación de estos estudios en el Espacio Europeo de Educación Superior para abordar después un análisis de la titulación con el fin de encontrar semejanzas y diferencias que ofrezcan resultados óptimos para llevar a cabo la última fase de la investigación, en la que se pretende aportar la posibilidad de una mejora en la elaboración de programas, en el contexto universitario, en el ámbito de esta área de conocimiento. Se ha llevado a cabo una investigación de carácter exploratorio y descriptivo, puesto que trata de describir y presentar una disciplina novedosa y poco estudiada hasta el momento. Para ello, se ha utilizado la triangulación (combinación de metodología cualitativa y cuantitativa) siguiendo los razonamientos metodológicos establecidos por R.D. Wimmer y J.R. Dominick (1996: 51).

Palabras clave: Espacio Europeo de Educación Superior (EEES); protocolo; organización de eventos; Bolonia; cuestionario.

\section{[en] Teaching of event management in the European Higher Education Area: comparative analysis of the curriculum}

\begin{abstract}
The study of protocol research and event management has only just recently been introduced in the Spanish University context. In fact, it is currently offered in only five universities. However, it is a field that has a long university tradition in Europe and the rest of the world. With the intention of finding similarities and differences between all university programs in Europe, this article assess the current situation of these studies in the European Higher Education Context in order to be able to offer optimal results to carry out the last phase of the research, which aims to provide an improvement in the development of programs in the university context, as part of this subject area. An explorative and descriptive research, which attempts to describe and present a new discipline scarcely studied so far, has been conducted. The method of choice was triangulation (a combination of the qualitative and quantitative methodology) following the methodological reasoning established by R.D. Wimmer and J.R. Dominick (1996: 51).
\end{abstract}

Keywords: European Higher Education Area (EHEA); protocol; event management; Bologna; questionnaire.

Sumario. 1. Introducción. 2. Metodología. 3. Resultados. 4. Conclusiones 5. Referencias bibliográficas.

\footnotetext{
$1 \quad$ Universidad de Alcalá de Henares.

E-mail: jc.herrero@uah.es

2 Escuela Universitaria de Turismo Felipe Moreno (Centro adscrito a la Universidad de las Islas Baleares)

E-mail: mperello@etb-baleares.es
} 
Cómo citar: Herrero, Julio César y Perelló Roselló, María del Mar (2017): “La enseñanza del protocolo y la organización de eventos en el espacio europeo de educación superior: análisis comparado de los planes de estudio", en Estudios sobre el Mensaje Periodístico 23 (1), 437-455.

\section{Introducción}

El camino hacia la construcción del Espacio Europeo de Educación Superior (EEES) se inició con la firma de la Declaración de la Sorbona (1998) y de la Declaración de Bolonia (1999). Estaba previsto que todos los cambios, lo que implicaba la transformación de los planes de estudio, hubiesen culminado en el año 2010. Pero no todos los países, incluido España, han completado sus reformas, ya que se ha tardado en comprender la esencia del EEES. Se trata del comienzo de un proceso que detectó las carencias de los sistemas educativos europeos y propuso una serie de reformas para igualar la educación superior en todos los países.

El modelo del EEES se ha ido complementando tras las sucesivas reuniones de los ministros de educación en diferentes ciudades europeas (Bucarest, 2012; Budapest-Vienna, 2010; Bergen, 2005; Berlín, 2003; Londres, 2007; Lovaina, 2009; Praga, 2001) y ha centrado su actuación en la construcción de un sistema de educación superior entendible y semejante, basado en un marco común de cualificaciones con la implantación del Suplemento Europeo al Título, la adopción de una escala de titulaciones en tres niveles (Grado, Máster y Doctorado) con un sistema de créditos compartido, el European Credit Transfer System (ECTS), y una educación centrada en el alumno que le permita una movilidad libre de trabas en todo el EEES.

Haug y Mora (2011: 27) respaldan que el 'Proceso Bolonia' supone un diálogo entre los gobiernos, instituciones de educación superior y los propios estudiantes implicados, cuyo objetivo común es apostar por la movilidad, la competitividad internacional, la empleabilidad, el desarrollo de la educación superior, y la conciliación entre los sistemas que garantizan la calidad de la enseñanza en cada uno de los países.

El Proceso de Bolonia ha reformado el panorama de la educación superior en Europa. Todos los países han llevado a cabo cambios significativos que han motivado la creación del Espacio Europeo de Educación Superior y posibilitado una educación superior que responde a las demandas de la sociedad. Las estructuras de educación superior han cambiado, se han desarrollado sistemas de garantía de calidad y se han puesto en marcha mecanismos para la movilidad de estudiantes y profesores.

Se trata de la gran oportunidad de la universidad española para equipararse con el resto de Europa ofreciendo una formación de calidad y una oferta de títulos que le permita ser competitiva. En este sentido, no extraña la reciente implantación de los estudios oficiales en materia de Protocolo y Organización de Eventos en España.

Bolonia representa el marco ideal para desarrollar unos estudios de Grado, cuya demanda consta desde 1995 por parte de los profesionales del ámbito, quienes hicieron la primera petición oficial en el I Congreso Internacional de Protocolo, en Oviedo. Solicitaban en las conclusiones finales el reconocimiento oficial de los estudios de Protocolo por parte del Ministerio. En la actualidad, se ofertan cinco titulaciones en España - el Grado de Protocolo y Organización de Eventos en la Universidad Camilo José Cela (UCJC) desde 2010, el Grado de Protocolo, Organización de 
Eventos y Relaciones Institucionales en la Universidad Miguel Hernández (UMH) desde 2012, el Grado en Protocolo y Organización de Eventos en la Universidad Europea de Madrid desde 2013, el Grado en Protocolo, Organización de Eventos y Comunicación Corporativa en la Universidad Rey Juan Carlos y el Grado en Protocolo y Organización de Eventos en la Escuela Universitaria de Turismo 'Felipe Moreno' (Centro Adscrito a la Universidad de las Islas Baleares) desde 2015 - frente a las 142 detectadas en el resto del EEES con la misma denominación, Event Management (término europeo para denominar los estudios de Protocolo y Organización de Eventos). La tradición de los estudios de Grado en este ámbito está ampliamente extendida en Europa, especialmente en Reino Unido, donde, los currículos son revisados y actualizados constantemente, según las necesidades del mercado.

Tras un paseo por la 'historia', se ha podido comprobar que el protocolo engloba una de las profesiones más antiguas, sirviendo toda su labor para engrandecer o defenestrar las relaciones entre diferentes países. Los estudios de Protocolo y Organización de Eventos abarcan el asesoramiento, planificación y organización de eventos en instituciones o entidades, tanto a nivel nacional como internacional, además de la aplicación de las normas y tradiciones actuales del ceremonial en provecho de la sociedad.

Así pues, el interés académico de estos estudios radica en el conocimiento de la disciplina y de sus relaciones de poder a lo largo de la historia de nuestra sociedad, conociendo la tradición de las distintas culturas de la humanidad, las relaciones sociales, las normas y reglas, las tradiciones con el objetivo de ahondar en el saber de las relaciones internacionales. El cumplimiento de este propósito, junto con su puesta en escena, no sería sino todo un sustento para menguar las dificultades interculturales y entre estados. A todo ello debemos sumar, aquello que conlleva el concepto moderno del protocolo. Nos referimos al conocimiento de las artes gráficas, de la creatividad del mensaje, de la escenografía, entre otras.

Si bien existe larga tradición en España en la formación en Protocolo y Organización de Eventos, se trataba de estudios no oficiales. El primer contacto de los estudios en este ámbito con la universidad española se remonta al año 1997 cuando, de forma paralela, la Universidad de Granada (UGR) y la Universidad Miguel Hernández de Elche implantaron el Título Propio en Protocolo y Relaciones Institucionales. Esta formación se elaboró pensando en las licenciaturas existentes y contaba con el mismo sistema de créditos y tipología de asignaturas. De esta forma, este paralelismo ofreció la posibilidad de formar profesionales que atendían a las necesidades del sector. Un sector, el de los eventos, como afirma Izquierdo (2012), "con muchísimo futuro [...]. Somos un sector fuerte. Los informes demuestran que los eventos se afianzan como una herramienta de comunicación, respondiendo mejor a la crisis que el marketing [...]. La importancia del sector de eventos en nuestro país queda patente en su aportación al PIB, estimada en un 7\%".

Con la oficialización de los estudios a nivel de grado y de postgrado de Protocolo y Organización de Eventos, y habida cuenta del interés académico, profesional y científico que ello conlleva, es necesario aportar a esta disciplina investigaciones científicas y trabajo de campo.

La investigación teórica en protocolo y organización de eventos se ha realizado desde el plano histórico, el jurídico y el comunicológico. Éste último, muestra el protocolo como transmisor de la imagen de una organización y, por lo tanto, de su identidad. No son pocas las investigaciones científicas que han visto la luz en forma de tesis doctoral dejando patente que sí existe un interés académico por el protocolo. 
El crecimiento constante de publicaciones científicas periódicas: la llegada de revistas en las que se descubren trabajos de profesionales de la organización de eventos, junto con los trabajos de reputados periodistas, antropólogos, psicólogos, entre otros; la mejora de la calidad de los trabajos publicados; la organización de congresos y eventos internacionales (destacamos el Congreso Internacional de Protocolo, que se celebra anualmente, y los congresos que respalda la Association for Events Management Education [AEME], que integra a más de 100 universidades del mundo) demuestran la evolución del Protocolo y Organización de Eventos, y de las inquietudes de los profesionales del ámbito.

Asimismo, la mayoría de las publicaciones científicas existentes en el ámbito manifiestan una clara relación con las divulgaciones del ámbito universitario, quedando patente el ferviente apoyo por parte de las instituciones de educación superior para la investigación, el análisis y la difusión en el protocolo y la organización de eventos. De igual forma, el interés progresivo de los países integrantes del Espacio Europeo de Educación Superior y de países americanos se alzan como aval por el interés académico que esta disciplina promueve en el mundo universitario, ratificado por una amplia bibliografía publicada en nuestro país y en el extranjero.

Cabe destacar en este punto, los precedentes en cuestión de investigaciones y estudios similares al que proponemos. Desde la puesta en marcha del 'Proceso Bolonia' han proliferado las publicaciones en torno al nuevo escenario educativo, tanto en el ámbito de la Comunicación como en otras disciplinas. Es interesante observar los diferentes tipos de estudios que se han llevado a cabo y la metodología que han usado, pues han servido para diseñar las diferentes fases de la investigación que nos ocupa.

De la revisión bibliográfica realizada podemos destacar diferentes autores y trabajos. Joan María Senent trabajó en el ámbito de la pedagogía realizó diversos análisis comparados de titulaciones en Europa buscando conocer las situaciones de los diferentes países para establecer tendencias generales en Europa; la investigación de la Red ALFA EVEDMUS (2008) tenía como objetivo indagar en los planes de estudio actualmente implantados de Formación del Profesorado en Educación Musical en Europa y América Latina para producir conocimiento de sus condiciones, causa y formas en la construcción del conocimiento musical y educativo que se está realizando en la práctica; del trabajo de Francisco Javier Blanco y $\mathrm{M}^{\mathrm{a}}$ José Latorre (2010) es interesante el método (cuestionario) utilizado en su estudio que pretende la creación de un programa de formación para el profesorado universitario a partir del estudio de necesidades e intereses de éstos; y el análisis comparativo de los planes de estudios de la licenciatura de la Universidad Autónoma de Sinaloa (México) y el Grado de la Universidad Politécnica de Madrid (España) de Francisco José Álvarez y Clemente García (2010), que integra un estudio comparado entre titulaciones de distintos países.

Es interesante señalar en este punto, que son cada día más frecuentes los trabajos precedentes, en el ámbito de la Comunicación, área que nos ocupa en nuestra investigación. Sotelo y Marcos (2007) reflexionaron sobre la enseñanza del periodismo en el contexto del EEES; Gallareta (2008), presentó un análisis comparativo de los planes de estudio de la Licenciatura de Publicidad de México, Argentina y Colombia, y del mismo modo lo hizo López (2010) con la formación de periodistas en Brasil, Puerto Rico, Portugal y España; en 2009, García y Martínez, mostraron las competencias que deberían adquirir los estudiantes de grado en Periodismo en el EEES y 
cómo podían incentivarse éstas a través de las nuevas tecnologías; Barranquero y Redondo (2009) y Sánchez (2013) estudiaron la licenciatura y el Grado en Periodismo, en un análisis comparado antes y después de la implantación del 'Plan Bolonia'.

Nuestro estudio se centra en el análisis comparado de los planes de estudios de Organización de Eventos - y Protocolo- en el EEES con el fin de contribuir a mejorar la educación y la formación, en el contexto universitario, en el ámbito del Protocolo y la Organización de Eventos.

Los objetivos específicos son: analizar la situación de los estudios de grado en Protocolo y Organización de Eventos en el marco actual de la educación universitaria; detectar qué países miembros de la Unión Europea han incluido en las universidades estudios de grado de esta disciplina; desarrollar un análisis de la titulación en cada uno de los países con el fin de encontrar semejanzas y diferencias que ofrezcan resultados óptimos para llevar a cabo la última fase de la investigación: plasmar la prospectiva ideal del currículo del Grado de Protocolo y Organización de Eventos. Con todo ello se pretende contribuir al planteamiento de un registro actualizado de las universidades y planes de estudio de Protocolo y Organización de Eventos y proponer una herramienta para facilitar el proceso de los programas de movilidad de estudiantes en el marco del Espacio Europeo de Educación Superior.

Algunas de las hipótesis que planteamos son: 1) las titulaciones ofertadas en España muestran un carácter más generalista en el ámbito de la Comunicación frente a las titulaciones ofertadas en el resto del EEES, más centradas en el ámbito de la empresa, el turismo y la hostelería, y con una estructura de materias mucho más específica; 2) la duración de las titulaciones en España es mayor que en la mayoría del resto de países; 3) al contrario que la universidad española, en el resto del EEES, las instituciones poseen mayor libertad para presentar sus programas.

\section{Metodología}

La investigación realizada es de carácter exploratorio y descriptivo (Sierra Bravo, 1992:33), puesto que trata de describir y presentar una disciplina novedosa y poco estudiada hasta el momento. Wimmer y Dominick (1996: 51) apuntan que diferentes metodólogos han advertido que tanto la metodología cuantitativa como la cualitativa son importantes en la investigación de un fenómeno concreto por lo que, recientemente, se apuesta por la triangulación, término utilizado por estos autores para describir el uso combinado de ambas metodologías en una investigación. Es el caso del trabajo que nos ocupa.

En primer lugar, y en consonancia con la novedad de la investigación como ya se ha indicado, se optó por consultar a un informante clave experto en el objeto a investigar: estudios superiores en protocolo y organización de eventos. Como indica Ander-Egg (2003: 307), de los cinco tipos de informantes clave que se dan, escogimos un experto de reconocido prestigio en relación con el tema de investigación que, además, es un profesional en la disciplina que dispone de información relevante al respecto. A través de esta técnica cualitativa, pudimos conocer el estado de la cuestión. Por un lado, se realizó una búsqueda de las universidades con estudios en protocolo y organización de eventos en el EEES y se elaboró un listado con las 142 titulaciones detectadas, y por otro lado, se recabó bibliografía adecuada para establecer el contexto y marco teórico. Además, el informante clave nos ayudó a conformar 
un grupo de expertos para ser entrevistados con el fin de afianzar tanto estado de la cuestión como la técnica escogida para la recolección de datos: el cuestionario (método cuantitativo).

En la línea de González (1997), se diseñó un cuestionario ad hoc y, posteriormente, se acreditó mediante la técnica de 'validación de expertos'. El método de validación de expertos se basa en la consulta a personas que poseen grandes conocimientos sobre el objeto de estudio para la validar la propuesta que se les presenta habida cuenta de sus investigaciones, experiencia, etc. (Cruz, 2006: 10).

Para elaborar un 'borrador inicial', se realizó una revisión bibliográfica de los estudios anteriormente señalados vinculados al proceso de implantación de los estudios superiores en el nuevo marco europeo.

$\mathrm{El}$ 'borrador inicial' incluyó una serie variables que podrían denominarse comunes para el análisis de una titulación, independientemente de la disciplina que se quiera analizar (Tabla 1). Y siguiendo los criterios exigidos por la Agencia Nacional de Evaluación de la Calidad y Acreditación (ANECA, 2012), para la verificación de títulos oficiales se incluyeron variables más concretas para obtener información específica sobre un plan de estudios (Tabla 2)

Tabla 1. Variables incluidas en el 'borrador inicial' del cuestionario. Elaboración propia.

\begin{tabular}{|c|c|}
\hline $\mathbf{N}^{\mathbf{0}}$ & Variable \\
\hline 1 & Denominación de los estudios \\
\hline 2 & Centro en el que se imparten \\
\hline 3 & Años de duración \\
\hline 4 & Créditos ECTS totales \\
\hline 5 & Año de implantación \\
\hline 6 & Año de adaptación a Bolonia \\
\hline 7 & Rama de conocimiento a la que pertenece \\
\hline 8 & Área de estudio a la que pertenece \\
\hline 9 & Continuación de los estudios \\
\hline
\end{tabular}

Tabla 2. Variables sobre los planes de estudio. Elaboración propia.

\begin{tabular}{|c|c|}
\hline $\mathbf{N}^{\mathbf{0}}$ & Variable \\
\hline 10 & Modalidad en la que se imparten los estudios \\
\hline 11 & Idioma en que se imparten la titulación \\
\hline 12 & Créditos de Formación Básica \\
\hline 13 & Créditos Obligatorios \\
\hline 14 & Itinerarios o menciones (si se incluyen) \\
\hline 15 & Créditos de Prácticas Externas \\
\hline 16 & Créditos de Trabajo Fin de Grado \\
\hline
\end{tabular}

Asimismo, se añadieron variables relativas a los datos sociodemográficos del encuestado.

Para concretar las variables específicas de los estudios de Protocolo y Organización de Eventos y con la ayuda del informante clave, se entrevistó a ocho docentes 
expertos en la materia, acerca de los contenidos curriculares imprescindibles que debe contener la formación. Las aportaciones realizadas por los expertos fueron:

- Cualquier estructura de un plan de estudios en Organización de Eventos pasa por todo lo que tenga que ver con las técnicas de organización, el protocolo en la empresa, el protocolo no oficial, la producción y las técnicas escenográficas, la legislación y el ceremonial, las relaciones públicas, los actos especializados, el protocolo internacional y las tecnologías.

- Materias como marketing aplicado, la organización de eventos e idiomas deben incluirse y deben tener carácter obligatorio.

- Es adecuado incluir la variable área de estudio ya que la titulación puede estar enfocada a la empresa, a la comunicación, a la ingeniería, etc.

- En cuanto a continuación de los estudios, argumentaron que sería interesante incluir doctorado, además de estudios de Máster.

- Recomendaron no incluir en el cuestionario el año de adaptación a Bolonia, ya que quien tenga que responder puede automatizar la respuesta según lo que hay-

- Consideraron que sería interesante incluir una pregunta sobre la inserción laboral que puede aportar datos interesantes para un posterior estudio.

Uniendo las aportaciones y las variables ya existentes, se ordenaron los ítems y se construyó el cuestionario final, y se sometió a la validación de un grupo de expertos del ámbito del protocolo y la organización de eventos, de la educación superior y de la estructuración y evaluación de planes de estudio.

Se seleccionó un panel de once expertos, de los que se tuvo que descartar a dos ya que que sus resultados no llegaron a tiempo para el análisis. En la tabla 3, se muestran, las características del panel.

Tabla 3. Panel de expertos. Elaboración propia.

\begin{tabular}{|c|l|l|l|c|}
\hline $\mathbf{N}^{\mathbf{0}}$ & \multicolumn{1}{|c|}{ Puesto de trabajo actual } & $\begin{array}{c}\text { Calificación } \\
\text { profesional }\end{array}$ & Categoría docente & $\begin{array}{c}\text { Años de } \\
\text { experiencia }\end{array}$ \\
\hline 1 & Directora de Ordenación Académica & Doctora & Profesora agregada & 11 \\
\hline 2 & Profesor & Máster & Profesor asociado & 12 \\
\hline 3 & Director de Grado & Máster & Profesor Director & 24 \\
\hline 4 & Profesora & Titulado Superior & Profesor asociado & 3 \\
\hline 5 & Secretario General & Doctora & Profesora asociada & 13 \\
\hline 6 & Profesora & Máster & Profesora asociada & 34 \\
\hline 7 & Directora General & Titulada Superior & $\begin{array}{l}\text { Profesora } \\
\text { colaboradora }\end{array}$ & 12 \\
\hline 8 & Profesora & Doctor & Profesor agregado & 38 \\
\hline 9 & Vicerrector de Ordenación Académica & & 34 \\
\hline
\end{tabular}

Cada experto se sometió a una autoevaluación previa a la validación del instrumento con el fin de valorar el nivel de competencia respecto al tema de investigación. La metodología aprobada en 1971 por el Comité Estatal para la Ciencia y la Técnica de la antigua URSS para la elaboración de pronósticos científico-técnicos, que perfeccionó Cruz (2006: 17) fue el instrumento de medida. 
En esta metodología la competencia se determina por un coeficiente (k). Éste se calcula por el coeficiente del nivel de conocimientos sobre el tema investigado $\left(\mathrm{k}_{\mathrm{c}}\right)$ más (semisuma) la medida de las fuentes de argumentación $\left(\mathrm{k}_{\mathrm{a}}\right)$. Para conocer el nivel de conocimientos sobre el tema el candidato debe realizar una autoevaluación utilizando una escala de 0 a 10; para obtener el resultado de $\mathrm{k}_{\mathrm{a}}$, debe llevarse a cabo otra autoevaluación, pero en este caso atendiendo a seis fuentes de argumentación diferentes (se realiza completando una tabla con escala tipo Likert).

Se comprobó que seis de los nueve expertos alcanzaron un nivel alto de competencia, ya que el punto de corte se fijó en 0,7 , examinando las características del panel (Tabla 4).

Tabla 4. Evaluación de la competencia de los expertos. Elaboración propia.

Kc: Coeficiente de conocimiento | Ka: Coeficiente de argumentación | K: Coeficiente de competencia

\begin{tabular}{|c|c|c|c|c|}
\hline $\mathbf{N}^{\mathbf{0}}$ & $\mathbf{K c}$ & $\mathbf{K a}$ & $\mathbf{K}$ & Evaluación \\
\hline 1 & 0,9 & 0,79 & 0,84 & Alto \\
\hline 2 & 0,8 & 0,66 & 0,73 & Medio \\
\hline 3 & 1 & 0,96 & 0,98 & Alto \\
\hline 4 & 0,8 & 0,84 & 0,82 & Alto \\
\hline 5 & 0,8 & 0,8 & 0,8 & Alto \\
\hline 6 & 0,7 & 0,96 & 0,83 & Alto \\
\hline 7 & 0,9 & 0,83 & 0,86 & Alto \\
\hline 8 & 0,7 & 0,75 & 0,725 & Medio \\
\hline 9 & 0,9 & 0,62 & 0,76 & Medio \\
\hline
\end{tabular}

La metodología empleada por el Comité Estatal para la Ciencia y la Técnica de la antigua URSS y perfeccionada por Cruz (2006: 17), se ha implementado con éxito en otros ámbitos como la economía (especialmente en el turismo) y en la educación.

Para validar el cuestionario, cada experto debía valorar la adecuación de cada ítem con respecto al objetivo del instrumento. El cuestionario inicial constaba de 32 ítems. Además, se incluyó una pregunta abierta, donde el experto podía indicar las apreciaciones, sugerencias o recomendaciones que estimaba oportunas. 15 preguntas de 32 fueron valoradas como muy adecuadas y 17 como bastante adecuadas.

El cómputo global de respuestas obtenidas nos indicó que en el 70,48\% de los casos se obtuvo una respuesta de 'ítem muy adecuado' y un $25 \%$ de 'ítem bastante adecuado'. La categoría 'nada adecuado' nunca fue seleccionada por ninguno de los expertos.

Cabe mencionar las recomendaciones de algunos de los expertos, que contribuyeron al ajuste del cuestionario para su versión definitiva, las cuales se presentan a continuación:

- Se recomendó añadir también, a partir de los ítems 28 ("la Universidad, ¿mide la tasa de graduación de los egresados en el Grado de Protocolo y Or- 
ganización de Eventos?”) y 30 (“la Universidad, ¿mide la inserción laboral de los egresados en el Grado de Protocolo y Organización de Eventos?"), las tasas de éxito y abandono de la titulación. No se han incluido estas preguntas, pues se consideran suficientes las tasas de graduación e inserción laboral y no se quiere correr el riesgo de enviar un cuestionario muy largo, susceptible de no ser respondido.

- Se consideró que los ítems 1 ("cargo que ocupa la persona que responde"), 2 ("categoría docente de la persona que responde"), 3 ("nombre de la Universidad o Institución”) y 32 (“¿podría facilitarnos su correo electrónico para un posterior contacto?") no tienen que ver con la investigación por lo que se categorizan de poco adecuados. Se han mantenido estos ítems considerando necesario saber quién contesta el cuestionario y tener la posibilidad de un segundo contacto.

- El ítem 21 ("indique si en el plan de estudios se contemplan las siguientes asignaturas. De la relación de asignaturas que se encuentran a continuación y que se incluyan en el plan de estudios indique si son de carácter básico [FB], obligatorio [OB] u optativo [OP]") resultó muy adecuado, pero tal vez se creyó necesaria una reformulación de la pregunta para facilitar su cumplimentación. Se ha reformulado la pregunta en la versión final ("indique si en el plan de estudios, se contemplan las siguientes asignaturas. De la relación de asignaturas que se encuentran a continuación y que se incluyan en el plan de estudios indique si son de carácter básico [FB], obligatorio [OB] u optativo [OP]").

- Se recomendó añadir las materias 'producción de eventos' y 'organización de eventos' en el ítem 21. En el cuestionario final, se han añadido estas asignaturas.

- Uno de los expertos consideró la posibles diferencias entre los estudios en España y el resto de países del EEES y marcó los ítems 13 ("el plan de estudios, ¿cuenta con créditos de Formación Básica? En caso afirmativo, indique la cantidad de créditos"), 15 (“¿contempla el plan de estudios itinerarios o especialidades?"), 16 ("indique la denominación de los itinerarios o especialidades"), 17 (“ ¿contempla el plan de estudios prácticas externas curriculares?"), 18 ("indique la cantidad de créditos de prácticas externas curriculares"), 20 (“¿Cómo se estructura el plan de estudios? Indiqué cómo se distribuye"), 28 ("la Universidad, ¿mide la tasa de graduación de los egresados en el Grado de Protocolo y Organización de Eventos?"), 29 (“¿cuál es la tasa de graduados de la titulación?"), 30 ("la Universidad, ¿mide la inserción laboral de los egresados en el Grado de Protocolo y Organización de Eventos?”), 31 (“¿cuál es la tasa de inserción laboral de la titulación?") como bastante adecuados, si bien manifestó necesaria una revisión de la normativa del marco europeo para facilitar la recogida y categorización de datos y evitar errores en la codificación. Tras una nueva revisión, se ha eliminado el ítem 20 con el fin de evitar confusiones en la categorización (de este modo, el ítem 21 del cuestionario final pasa a ser el 20).

Tras la validación de expertos, se elaboró la versión final del cuestionario (tabla 5) y se tradujo al inglés para su envío. 
Tabla 5. Cuestionario final. Elaboración propia.

\section{Versión en español}

1. Cargo que ocupa la persona que responde.

Haga clic aquí para escribir texto.

2. Categoría docente de la persona que responde.

Catedrático/a de universidad o escuela universitaria

Profesor/a asociado/a

Profesor/a contratado/a doctor/a

Profesor/a ayudante doctor/a

Profesor/a colaborador/a

Otro: Haga clic aquí para escribir texto.

3. Indique el nombre de la Universidad/Institución.

Haga clic aquí para escribir texto.

4. Indique la Facultad o Escuela en la que se imparten los estudios.

Haga clic aquí para escribir texto.

5. Indique la denominación de la titulación.

Haga clic aquí para escribir texto.

6. A qué rama de conocimiento está asociada la titulación?

Artes y Humanidades

$\checkmark$ Ciencias Sociales y Jurídicas

$\square$ Ciencias

$\square$ Ciencias de la Salud

Ingeniería y Arquitectura

7. ¿A qué área de estudio está asociada la titulación?

Artes y Humanidades

Educación

Ciencias Sociales

Economía y Empresa

Derecho

Salud

Ciencias

Ingeniería y Arquitectura

Otra: Haga clic aquí para escribir texto.

8. ¿Cuál es la duración de los estudios?

4 años

3 años

2 años

Otra: Haga clic aquí para escribir texto.

9. ¿Cuántos créditos completan el plan de estudios?

240 créditos ECTS

180 créditos ECTS

Otro: Haga clic aquí para escribir texto. 
10. Indique el año de implantación de los estudios

Haga clic aquí para escribir texto.

11. ¿En qué modalidad se imparten los estudios? (Si se ofrecen varias modalidades, indique cada una de ellas).

$\checkmark$ Presencial

Semipresencial

A distancia

Otra: Haga clic aquí para escribir texto.

12. ¿En qué idioma se imparte la titulación? (En el caso de que se imparta en varios idiomas, indicar cada uno de ellos).

Haga clic aquí para escribir texto.

13. El plan de estudios, ¿cuenta con créditos de Formación Básica? En caso afirmativo, indique la cantidad de créditos.

Haga clic aquí para escribir texto.

14. Indique la cantidad de créditos obligatorios que hay en el plan de estudios.

Haga clic aquí para escribir texto.

15. El plan de estudios, ¿cuenta con créditos optativos? En caso de que la respuesta sea afirmativa, indique la cantidad de créditos.

Haga clic aquí para escribir texto.

16. ¿Contempla el plan de estudios itinerarios o especialidades? (Si se contemplan, pero no son de carácter obligatorio, indicar opcional)

$$
\begin{aligned}
& \square \text { Sí } \\
& \text { No } \\
& \square \text { Opcionales }
\end{aligned}
$$

17. Indique la denominación de los itinerarios o especialidades que se incluyen en el plan de estudios. (Conteste está pregunta solo si hay indicado 'sí' o 'opcionales' en la pregunta anterior).

Haga clic aquí para escribir texto.

18. ¿Contempla el plan de estudios Prácticas Externas Curriculares?

$$
\begin{aligned}
& \square \text { Sí } \\
& \square \text { No } \\
& \square \text { Opcionales }
\end{aligned}
$$

19. Indique la cantidad de créditos de prácticas externas curriculares que hay en el plan de estudios. (Conteste está pregunta solo si hay indicado 'sí' o 'opcionales' en la pregunta anterior).

Haga clic aquí para escribir texto.

20. El plan de estudios, ¿incluye Trabajo Fin de Grado o Proyecto final? En caso afirmativo, indique la cantidad de créditos.

Haga clic aquí para escribir texto.

21. Indique si en el plan de estudios, se contemplan las siguientes asignaturas. (De la relación de asignaturas que se encuentran a continuación y que se incluyan en el plan de estudios indique si son de carácter básico [FB], obligatorio [OB] u optativo [OP]. 


\begin{tabular}{|c|c|c|c|}
\hline ASIGNATURAS & FB & OB & OP \\
\hline Técnicas de Organización de Eventos & $\square$ & $\square$ & $\square$ \\
\hline Empresa/ Gestión Empresarial & $\square$ & $\square$ & $\square$ \\
\hline Relaciones Públicas & $\square$ & $\square$ & $\square$ \\
\hline Marketing/ Marketing Aplicado & $\square$ & $\square$ & $\square$ \\
\hline Planificación de Eventos & $\square$ & $\square$ & $\square$ \\
\hline Industria de Eventos & $\square$ & $\square$ & $\square$ \\
\hline Seguridad y Legislación Comunitaria & $\square$ & $\square$ & $\square$ \\
\hline Técnicas escenográficas & $\square$ & $\square$ & $\square$ \\
\hline Relaciones Internacionales & $\square$ & $\square$ & $\square$ \\
\hline Eventos Empresariales & $\square$ & $\square$ & $\square$ \\
\hline Eventos Deportivos & $\square$ & $\square$ & $\square$ \\
\hline Eventos Académicos & $\square$ & $\square$ & $\square$ \\
\hline Eventos Turísticos & $\square$ & $\square$ & $\square$ \\
\hline Grandes Eventos & $\square$ & $\square$ & $\square$ \\
\hline Mercado de Reuniones, Ferias y Exposiciones & $\square$ & $\square$ & $\square$ \\
\hline Nuevas Tecnologías & $\square$ & $\square$ & $\square$ \\
\hline Tecnologías Aplicadas & $\square$ & $\square$ & $\square$ \\
\hline Instituciones Públicas y su ceremonial & $\square$ & $\square$ & $\square$ \\
\hline Restauración & $\square$ & $\square$ & $\square$ \\
\hline Idioma & $\square$ & $\square$ & $\square$ \\
\hline
\end{tabular}

22. Indique si en la relación del plan de estudios, se imparten asignaturas en un idioma distinto al oficial. (De la relación de asignaturas que se encuentran a continuación y que se incluyan en el plan de estudios indique si se dan en el idioma oficial, en otro idioma o en ambos idiomas)

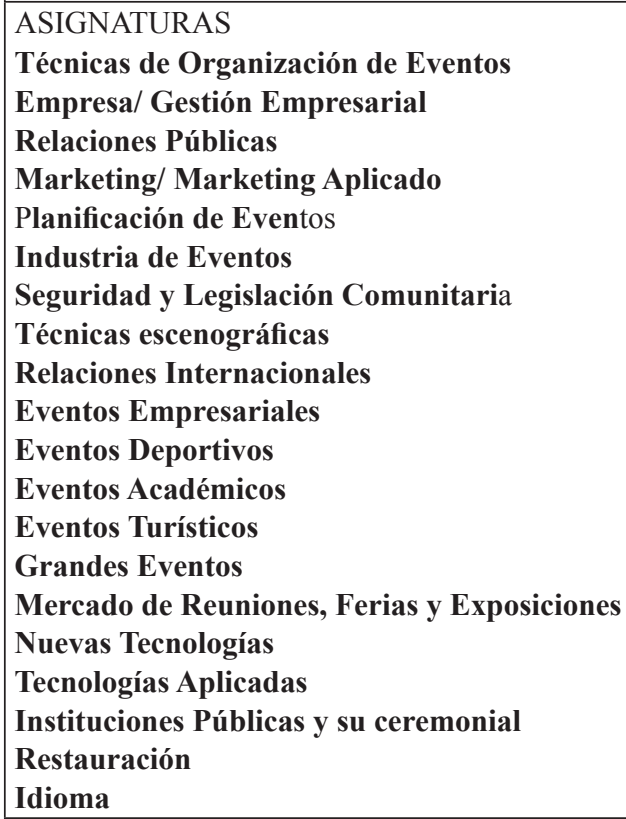

23. Si considera que el plan de estudios contempla algunas asignaturas de interés, que no se haya nombrado en la pregunta anterior, indíquelas a continuación.

Haga clic aquí para escribir texto. 
24. ¿Ofrece la Universidad estudios de postgrado (Máster) la disciplina de Protocolo y Organización
de Eventos?
$\square$ Sí
$\square$ No

25. ¿Cuál es la denominación de la o las titulaciones de Máster que ofrece? (Conteste está pregunta solo si hay indicado 'sí' en la pregunta anterior).

Haga clic aquí para escribir texto.

26. ¿Ofrece la Universidad programas de Doctorado en la disciplina de Protocolo y Organización de Eventos?

Sí

No

27. ¿Cuál es la denominación del o los programas de Doctorado que ofrece? (Conteste está pregunta solo si hay indicado 'sí' o en la pregunta anterior)

Haga clic aquí para escribir texto.

28. La Universidad, ¿mide la tasa de graduación de los egresados en el Grado de Protocolo y Organización de Eventos?

Sí

No

29. ¿Cuál es la tasa de graduación de los egresados? (Conteste está pregunta solo si hay indicado 'sí' o en la pregunta anterior)

Haga clic aquí para escribir texto.

30. La Universidad, ¿mide la inserción laboral de los egresados en el Grado de Protocolo y Organización de Eventos?

$\square$ Sí

$\square$ No

31. ¿Cuál es la tasa de inserción laboral de los egresados? (Conteste está pregunta solo si hay indicado 'sí' o en la pregunta anterior).

Haga clic aquí para escribir texto.

32. ¿Podría facilitarnos su correo electrónico para un posterior contacto? (Le agradeceríamos, en la medida de lo posible, que nos facilitase su correo electrónico por si necesitamos contactar con usted en relación a la investigación).

Como ya se ha señalado anteriormente, el alcance internacional que ha adquirido la investigación exigió una labor de localización de contactos para responder al cuestionario. Para ello, se consultaron las páginas webs de las principales universidades en cada uno de los países integrantes del EEES y se creó una base de datos que contenía el nombre de la universidad, el país, el nombre y el correo electrónico de la persona de contacto, la denominación de la titulación y la URL consultada.

La base de datos contenía 142 contactos posibles y el cuestionario se envió a todos estos contactos a través de Internet creando un formulario de Google Drive, controlando el acceso y la evolución mediante correos electrónicos de invitación a responder el mismo. Finalmente, se realizaron cuatro envíos de correos electrónicos y fueron 31 los contactos que respondieron correctamente y que han podido incluirse en el estudio final.

El obstáculo idiomático ha sido clave en la pérdida sustancial de muestra. Aun así, la investigación ha podido continuar su curso, contando con la participación de titulaciones de grado de diferentes países (Tabla 6). 
Tabla 6 Titulaciones incluidas en el estudio final. Elaboración propia.

\begin{tabular}{|c|c|c|}
\hline País & Universidad/ Centro & Titulación \\
\hline \multirow{7}{*}{ Alemania } & $\begin{array}{l}\text { Hochschule fuer Internationales } \\
\text { Management Heidelberg }\end{array}$ & $\begin{array}{l}\text { International Business with Event } \\
\text { Management Bachelor of Arts }\end{array}$ \\
\hline & International School of Management & B.A. Tourism and Event Management \\
\hline & Macromedia University of Applied Sciences & Sport and Event Management \\
\hline & BBW Hochschule & $\begin{array}{l}\text { Tourism and Event Management Bachelor } \\
\text { of Arts }\end{array}$ \\
\hline & $\begin{array}{l}\text { Fachhochschule des Mittelstand, Bielefeld, } \\
\text { Germany }\end{array}$ & $\begin{array}{l}\text { Bachleor of Arts in Event Management and } \\
\text { Entertainment }\end{array}$ \\
\hline & EBC-Hochschule Campus Berlin & $\begin{array}{l}\text { Tourism \& Eventmanagement BA, } \\
\text { International Bachelor of Arts }\end{array}$ \\
\hline & IUBH -University of Applied Sciences & International Event Management \\
\hline Austria & UAS Kufstein Tirol Austria & Sports Culture \& Events Management \\
\hline Dinamarca & University of Southern Denmark & $\begin{array}{l}\text { Bachelor in Economics and Business } \\
\text { Administration - Sport and Event } \\
\text { Management }\end{array}$ \\
\hline \multirow{2}{*}{ España } & Universidad Camilo José Cela & $\begin{array}{l}\text { Grado en Protocolo y Organización de } \\
\text { Eventos }\end{array}$ \\
\hline & Universidad Miguel Hernández & $\begin{array}{l}\text { Grado en Organización de Eventos, } \\
\text { Protocolo y Relaciones Institucionales }\end{array}$ \\
\hline \multirow[b]{2}{*}{ Francia } & Université Nice Sophia-Antipolis & $\begin{array}{l}\text { Licence Professionnelle Communicaion } \\
\text { Evenementielle }\end{array}$ \\
\hline & Université de Franche-Comté & \begin{tabular}{|l|} 
Licence Professionnelle "Marketing et \\
communication des Organisations, du \\
Spectacle, de l'Evénementiel et des Loisirs"
\end{tabular} \\
\hline \multirow{3}{*}{ Irlanda } & Dublin Institute of Technology & B.Sc. Event Management \\
\hline & American College Dublin & BA in Event Management \\
\hline & Galway Mayo Institute of Technology & $\begin{array}{l}\text { Bachelor of Business in Event Management } \\
\text { and Public Relations }\end{array}$ \\
\hline Islandia & UGLA Holar University College & Event Management \\
\hline Portugal & Instituto Politécnico de Leiria & Gestao de Eventos \\
\hline \multirow{13}{*}{ Reino Unido } & University of Chester & BA Events Management \\
\hline & Glasgow Caledonian University & BA (Hons) International Events Management \\
\hline & Arts University Bournemouth & Creative Events Management \\
\hline & University of Central Lancashire & BA (Hons) Events Management \\
\hline & University of Winchester & BA (Hons) Events Management \\
\hline & Glyndwr University & BA Festival and Event Industry \\
\hline & University of Greenwich & BA Events Management \\
\hline & University of West London & BA (Hons) Events Management \\
\hline & BIMM Group & BA (Hons) Events Management \\
\hline & UCS - University Campus Suffolk & BA (Hons) Events Management \\
\hline & Bournemouth University & BA (Hons) Events Management \\
\hline & University of Derby & Bachelor of Arts in Events Management \\
\hline & Bucks New University & BA Music and Live Event Management \\
\hline
\end{tabular}


Una vez recibido el formulario cumplimentado de las 31 universidades se revisaron las respuestas y se volvió a contactar, esta vez individualmente, para la resolución de dudas y cerrar una ficha con cada titulación. En este punto, dos fueron los aspectos clave a resolver en esta fase de investigación. Por un lado, se unificó la variable crédito de formación básica y crédito obligatorio puesto que, salvo en España (y en algún caso concreto en Alemania), los sistemas educativos europeos diferencian entre créditos obligatorios y opcionales, sin hacer especificaciones dentro de estos dos tipos; por otro lado, se consultó la existencia de algún órgano regulador y revisor (como ANECA en España) en cada uno de los países. Las universidades enviaron, si era el caso, la agencia u organismo pertinente.

Tras estas consultas, el cuestionario final sufrió pequeñas modificaciones en las preguntas relativas al tipo de créditos.

Una vez recabados todos los datos, se ha utilizado el software de análisis estadístico Statistical Package for the Social Sciences (SPSS) para procesar todos los datos obtenidos.

\section{Resultados}

A continuación procedemos a mostrar los resultados obtenidos tras el análisis de los datos proporcionados por las universidades participantes.

Podemos decir que el $66 \%$ de las titulaciones analizadas pertenecen a la rama de conocimiento de Ciencias Sociales y Jurídicas frente a un 31\% que se incluirían en la rama de Artes y Humanidades. Tan solo una de las titulaciones analizadas se incluye en la rama de Ciencias (BA Tourism \& Eventmanagement de la EBCHochschule de Berlin).

Con el fin de especificar el campo de estudio de cada una de las titulaciones, además de la rama de conocimiento en la que se encuentra incluido el título, se solicitó el ámbito específico de cada uno. En este sentido, el campo de la economía y la empresa es el que más titulaciones recoge. Sorprende que tan solo un $6 \%$ sean del ámbito del turismo, tradicional en este tipo de estudios.

En la línea con la tendencia propuesta por la implantación del Espacio Europeo de Educación Superior la mayoría de titulaciones tienen una duración de 3 años (23) frente a cinco de 4 años. Se han detectado cuatro casos especiales con una duración diferente a las típicas. Del mismo modo, predominan los estudios de 180 créditos ECTS frente a los de 240 créditos ECTS.

De las materias y asignaturas incluidas en el cuestionario para analizar y valorar en qué medida existen similitudes en los planes de estudio podemos decir que todas las titulaciones analizadas incluyen gran parte las materias que el panel de expertos indicó como imprescindibles.

En este sentido, la siguiente tabla muestra, el porcentaje de titulaciones que incluyen cada una de las materias y si lo hacen como créditos obligatorios u optativos. 
Tabla 7. Materias incluidas en los planes por tipo. Elaboración propia.

\begin{tabular}{|l|c|c|c|c|}
\hline \multirow{2}{*}{\multicolumn{1}{|c|}{ Materia }} & \multicolumn{4}{c|}{ Carácter } \\
\cline { 2 - 5 } & \multicolumn{2}{|c|}{ Obligatoria } & \multicolumn{2}{c|}{ Optativa } \\
\hline Técnicas de Organización de Eventos & $96,77 \%$ & 30 & $3,23 \%$ & 1 \\
\hline Empresa/Gestión Empresarial & $96,77 \%$ & 30 & $3,23 \%$ & 1 \\
\hline Relaciones Públicas & $61,29 \%$ & 19 & $16,13 \%$ & 5 \\
\hline Marketing/ Marketing Aplicado & $93,55 \%$ & 29 & $6,45 \%$ & 2 \\
\hline Planificación de Eventos & $90,32 \%$ & 28 & $6,45 \%$ & 2 \\
\hline Industria de Eventos & $83,87 \%$ & 26 & $6,45 \%$ & 2 \\
\hline Seguridad y Legislación Comunitaria & $74,19 \%$ & 23 & $9,68 \%$ & 3 \\
\hline Técnicas escenográficas & $41,94 \%$ & 13 & $12,90 \%$ & 4 \\
\hline Relaciones Internacionales & $41,94 \%$ & 13 & $12,90 \%$ & 4 \\
\hline Eventos Empresariales & $67,74 \%$ & 21 & $9,68 \%$ & 3 \\
\hline Eventos Académicos & $48,39 \%$ & 15 & $12,90 \%$ & 4 \\
\hline Eventos Turísticos & $51,61 \%$ & 16 & $16,13 \%$ & 5 \\
\hline Grandes Eventos & $67,74 \%$ & 21 & $3,23 \%$ & 1 \\
\hline Mercado de Reuniones, Ferias y Exposiciones & $61,29 \%$ & 19 & $6,45 \%$ & 2 \\
\hline Nuevas Tecnologías & $58,06 \%$ & 18 & $9,68 \%$ & 3 \\
\hline Tecnologías Aplicadas & $45,16 \%$ & 14 & $19,35 \%$ & 6 \\
\hline Instituciones Públicas y su ceremonial & $51,61 \%$ & 16 & $12,90 \%$ & 4 \\
\hline Restauración & $54,84 \%$ & 17 & $9,68 \%$ & 3 \\
\hline Idioma & $48,39 \%$ & 15 & $19,35 \%$ & 6 \\
\hline
\end{tabular}

Se puede observar con los resultados obtenidos que las materias como Técnicas de Organización de Eventos, Gestión Empresarial y Marketing, aparecen en todos los planes de estudio. Planificación de Eventos e Industria de Eventos se consideran materias muy importantes también y se reflejan en 30 y 28 titulaciones, respectivamente.

Cabe destacar que las materias más específicas de algún ámbito de la organización de eventos se ven reflejadas en menor medida. Esto se debe a los itinerarios de especialización y a la formación de carácter optativo que tiene cada titulación.

Es importante señalar, por otro lado, que los idiomas y las nuevas tecnologías no se oferten en la mayoría de titulaciones (hay diez titulaciones que no reflejan una o ambas materias). En el contexto del Espacio Europeo de Educación Superior, creemos que estas materias deberían reflejarse en todos los casos y con carácter obligatorio.

\section{Conclusiones}

Podemos concluir que, en opinión de los expertos consultados, el cuestionario diseñado para recabar datos para el análisis de los estudios de grado de Protocolo y Organización de Eventos en el EEES, ha sido un instrumento efectivo. La técnica empleada, la validación de expertos, ha otorgado resultados óptimos para la investigación, pues se ha obtenido mucha información, que no habría surgido con otras técnicas. 
Por el contrario, el obstáculo idiomático ha impedido contar con mayor número de participantes y en consecuencia la muestra se ha visto reducida a 31. Aun así, es importante señalar que la muestra recoge variedad en cuanto a los países que se han podido incluir en el estudio final (9), siendo además Reino Unido el que más titulaciones aporta, puesto que es un país pionero en la formación del ámbito de la organización de eventos.

Podemos afirmar que la hipótesis 1) las titulaciones ofertadas en España muestran un carácter más generalista en el ámbito de la Comunicación frente a las titulaciones ofertadas en el resto del EEES, más centradas en el ámbito de la empresa, el turismo y la hostelería, y con una estructura de materias mucho más específica, se cumple en parte; y la hipótesis 2) la duración de las titulaciones en España es mayor que en la mayoría del resto de países, se cumplen en su totalidad. En cuanto a la hipótesis 3) al contrario que la universidad española, en el resto del EEES las instituciones poseen mayor libertad para presentar sus programas, podemos decir que tras la consulta individual a cada uno de los participantes y en la línea con lo dispuesto sobre la garantía de la calidad (2012) en los diferentes países del EEES, cabe decir que la filosofía de los países en cuanto a los sistemas de calidad difiere. Así pues, algunos países cuentan con organismos responsables que tienen potestad para autorizar programas o asesoran a los gobiernos en estas decisiones; otros, en cambio, estos organismos tienen como único cometido asesorar a las instituciones. En el primer grupo, se encuentran España, Portugal, Alemania, Dinamarca. Por otro lado, Francia, Irlanda, Islandia, Reino Unido, cuentan con organismos únicamente de asesoramiento por lo que tienen mayor libertad en la elaboración de sus programas.

Esta investigación quiere contribuir a la investigación en el ámbito del Protocolo y la Organización de Eventos, en su perspectiva comunicológica y académica, aportando al desarrollo y evolución de un campo de estudio totalmente emergente. Además, los investigadores creen que el cuestionario diseñado podría, según la disciplina a analizar, ser útil en distintas investigaciones en el ámbito de la educación, más aun actualmente que empiezan a ser visibles los cambios suscitados por el "Proceso Bolonia".

\section{Referencias bibliográficas}

Agencia Nacional de Evaluación de la Calidad y Acreditación[ANECA] (16 de enero de 2012): Guía de apoyo para la elaboración de la memoria de verificación de títulos oficiales universitarios. En: http://www.aneca.es/content/download/12155/136031/file/verifica_guia_v04_120116. pdf [Consulta: 16 de mayo de 2013]

Álvarez Montero, Francisco José y García Gerardo, Clemente (2010): “Un pequeño análisis comparativo de los estudios superiores de Informática entre el noroeste de México y España: el caso de la UAS y de la UPM". Revista iberoamericana de Educación. Volumen 53, 5. http://www.rieoei.org/deloslectores/3719Alvarez.pdf [Consulta: 17 de noviembre de 2014]

Ander-Egg, Ezequiel (2003): Métodos y técnicas de investigación social: técnica para recogida de datos de información. Buenos Aires, Lumen.

Aróstegui, José Luis (2010): "Formación del profesorado de música: planes de estudio en Europa y América Latina". Revista de curriculum y formación del profesorado, 14 (2), $1-5$. 
Barranquero, Alejandro y Redondo, Félix (2009): “Análisis Comparado de los planes de Estudio de Periodismo antes y después de Bolonia”, en VV.AA.: Actas del I Congreso Internacional Latina de Comunicación Social. En:

http://www.revistalatinacs.org/09/Sociedad/actas/14barranquero.pdf [Consulta: 17 de noviembre de 2014]

Blanco Encomienda, Francisco Javier y Latorre Medina, María José (2010): “Elaboración de un cuestionario dirigido al profesorado universitario ante el área europea de educación superior". Contextos Educativos, 13, 43-56.

Cruz, Miguel (2006): "El método Delphi en las investigaciones educacionales". Cuba, Instituto Superior Pedagógico de Holguín.

Declaración de Bolonia (s.f.): Recuperado el 23 de abril de 2013, URL: http://www.eees.es/pdf/Bolonia_ES.pdf

Gallareta, Clara (2008): “Análisis comparativo de los planes de estudio de la Licenciatura de Publicidad de México, Argentina y Colombia”. Creación y Producción en Diseño y Comunicación, 15, 53.

García Avilés, José Alberto y Martínez Bonastre, Oscar (2009): “Competencias en la formación universitaria de periodistas a través de nuevas tecnologías". Revista de la Facultad de Ciencias Sociales y Juridicas de Elche, I (4), 239-250.

González, María José (1997): Metodología de la investigación. Técnicas de recolección de datos. Alicante, Aguaclara (Colección Amalgama).

Haug, Guy y Mora, José Ginés (2011): España y el Proceso de Bolonia, un encuentro imprescindible. Academica Europea de Ciencias y Artes.

Izquierdo, Paula (Diciembre de 2012): Entrevista con Paula Izquierdo, coordinadora de QueremosOrganizarEventos (QOE). (Conexo.net, Entrevistador), en:

http://www.nexotur.com/conexo/paula/izquierdo/importancia/sector/eventos/espana/ queda/patente/su/aportacion/al/pib/estimada/7/54438/ [Consulta: 23 de abril de 2013]

López García, Xose (2010): "La formación de los periodistas en el siglo XXI en Brasil, España, Portugal y Puerto Rico". Revista Latina de Comunicación Social, 65, 231-243. http://www.revistalatinacs.org/10/art2/896_Santiago/18_Xose.html [Consulta: 13 de abril de 2012] DOI: 10.4185/RLCS-65-2010-896-231-243.

Lozano Ascensio, Carlos y Vicente Mariño, Miguel (2010): “La enseñanza universitaria de las Teorías de la Comunicación en Europa y América Latina". Revista Latina de Comunicación Social, 65, 255-265, en:

http://www.revistalatinacs.org/10/art2/898_URJC/20_Lozano.html [Consulta: 13 de abril de 2012] DOI: 10.4185/RLCS-65-2010-898-255-265.

Ministerio de Educación, Cultura y Deporte (2012): El Espacio Europeo de Educación Superior en 2012: Informe sobre la implantación del Proceso Bolonia. Bruselas, Agencia Ejecutiva en el Ámbito Educativo, Audiovisual y Cultural.

Ministerio de Educación, Cultura y Deporte (s.f.): Registro de Universidades, Centros y Títulos (RUCT). https://www.educacion.gob.es/ruct/consultaestudios?actual=estudios [Consulta: 16 de noviembre de 2015]

Sánchez García, Pilar (2013): "Propuesta metodológica para el análisis comparado de los planes de estudio en la licenciatura y el grado de periodismo en la Universidad Española". II Congreso Nacional sobre Metodología de la Investigación en Comunicación, pp. $365-$ 382). Valladolid, Universidad de Valladolid.

Senent, José María (2004): Análisis comparado de los estudios superiores de educación en Europa. Programa de Convergencia Europea. Agencia Nacional de Evaluación de la Calidad y Acreditación. 
Senent, José María (2005): “Los estudios de pedagogía en Europa en el contexto de la implantación del proceso Bolonia y la situación de la Educación Comparada". Revista Española de Educación Comparada, 11, 95-133.

Sotelo González, Joaquín y Marcos Recio, Juan Carlos (2007): “Una reflexión sobre la metodología docente para la enseñanza del periodismo en el marco del Espacio Europeo de Educación Superior". Congreso Internacional La renovación de metodologías docentes en su adaptación al Espacio Europeo de Educación Superior. Valladolid: Universidad Miguel de Cervantes.

Universidad Camilo José Cela (2010): Memoria para la verificación del título de Grado de Protocolo y Organización de Eventos. Manuscrito no publicado.

Wimmer, Roger. D., y Dominick, Joseph. R. (1996): La investigación cientifica de los medios de comunicación: una introducción a sus métodos. Barcelona, Bosch.

Julio César Herrero es doctor en Periodismo por la Universidad Complutense de Madrid. Especializado en Comunicación y Marketing Político, ha sido el decano de la Facultad de Comunicación de la Universidad Complutense de Madrid. Es investigador del Instituto de Estudios Latinoamericanos de la Universidad de Alcalá. Es Fellow del Real Colegio Complutense en Harvard.

María del Mar Perelló Roselló es licenciada en Comunicación Audiovisual por la Universidad Camilo José Cela (UCJC) y Diploma de Estudios Avanzados (DEA) por la misma Institución. Actualmente, se encuentra en proceso de Tesis Doctoral, titulada "Análisis Comparado de los Estudios de Grado de Protocolo y Organización de Eventos en el Espacio Europeo de Educación Superior" en la Universidad de las Islas Baleares. 